\title{
Some Subclasses of a New Class of Analytic Functions under Fekete-Szegö Inequality
}

\author{
G.S. Rathore ${ }^{1}$, Gurmeet Singh ${ }^{2}$, ${ }^{*}$ Lokendra Kumawat $^{3}$, S.K. Gandhi ${ }^{4}$, Preeti Kumawat ${ }^{5}$ \\ 1.3.4.5 Department of Mathematics \& Statistics \\ Mohanlal Sukhadia University, Udaipur,(Raj) \\ ${ }^{2}$ Department of Mathematics \\ GSSDGS Khalsa College, Patiala( India) \\ Email: ganshayamrathore@yahoo.co.in ${ }^{1}$, meetgur111@gmail.com ${ }^{2}$ \\ lokendrakumawat@yahoo.co.in ${ }^{3}$, gandisk28@gmail.com ${ }^{4}$, \\ preeti.kumawat30@gmail.com ${ }^{5}$
}

\begin{abstract}
In this paper we have introduced a new class of analytic functions and its subclasses by using principle of subordination with power law and obtained sharp upper bounds of the functional $\left|a_{3}-\mu a_{2}^{2}\right|$ for the analytic function $\mathrm{f}(\mathrm{z})=\mathrm{z}+\sum_{\mathrm{n}=2}^{\infty} \mathrm{a}_{\mathrm{n}} \mathrm{z}^{\mathrm{n}},|\mathrm{z}|<1$ belonging to these classes.
\end{abstract}

Keywords- Bounded functions, Starlike functions, Inverse Starlike functions and Univalent functions

\section{INTRODUCTION}

Let $\mathcal{A}$ denote the class of analytic functions in the unit $\operatorname{disc} \mathbb{E}=\{z:|z|<1 \mid\}$ of the form.

$$
\begin{aligned}
& f(z)=z+\sum_{n=2}^{\infty} a_{n} z^{n} \\
& (1.1)
\end{aligned}
$$

Let $\mathcal{S}$ be the class of analytic functions of the form (1.1), which are analytic univalent in $\mathbb{E}$.

In 1916, (Bieber Bach [3], [4]) proved that $\left|a_{2}\right| \leq 2$ for the functions $f(z) \in \mathcal{S}$. In 1923, Löwner [2] proved that $\left|a_{3}\right| \leq 3$ for the functions $f(z) \in \mathcal{S}$..

With the known estimates $\left|a_{2}\right| \leq 2$ and $\left|a_{3}\right| \leq 3$, it was natural to seek some relation between $a_{3}$ and $a_{2}{ }^{2}$ for the class $\mathcal{S}$, Fekete and Szegö [5] used Löwner's [2] method to prove the following well known result for the class $\mathcal{S}$. Let $f(z) \in \mathcal{S}$, then

$\left|a_{3}-\mu a_{2}^{2}\right| \leq\left[\begin{array}{l}3-4 \mu, \text { if } \mu \leq 0 ; \\ 1+2 \exp \left(\frac{-2 \mu}{1-\mu}\right), \text { if } 0 \leq \mu \leq 1 ; \\ 4 \mu-3, \text { if } \mu \geq 1 .\end{array}\right.$

(1.2)

The inequality (1.2) plays a very important role in determining estimates of higher coefficients for some sub classes $\mathcal{S}$ (Chhichra[6], Babalola[1]).

We define the subclasses $\mathrm{S}^{*}$ and $\mathcal{K}$ of $\mathcal{S}$ in following manner

$S^{*}$ denote the class of univalent starlike functions $g(z)=z+\sum_{n=2}^{\infty} b_{n} z^{n} \in \mathcal{A}$ satisfying the condition $\operatorname{Re}\left(\frac{z g(z)}{g(z)}\right)>0, z \in \mathbb{E}$.

(1.3)

$\mathcal{K}$ denote the class of univalent convex functions $h(z)=z+\sum_{n=2}^{\infty} c_{n} z^{n} \in \mathcal{A}$ satisfying the condition $R e \frac{\left(\left(z h^{\prime}(z)\right)\right.}{h^{\prime}(z)}>0, z \in \mathbb{E}$.

(1.4)
We introduce the class $\mathcal{A}(\alpha, \beta)$ of functions $g(z)=z+$ $\sum_{n=2}^{\infty} b_{n} z^{n} \in \mathcal{A}$ satisfying the condition

$$
\begin{aligned}
& \operatorname{Re}\left[(1-\alpha-\beta) f^{\prime}(z)+\alpha \frac{z f^{\prime}(z)}{f(z)}+\beta \frac{\left\{z f^{\prime}(z)\right\}^{\prime}}{f^{\prime}(z)}\right]>0, z \\
& \quad \in \mathbb{E} \\
& \text { i.e. } \quad(1-\alpha-\beta) f^{\prime}(z)+\alpha \frac{z f^{\prime}(z)}{f(z)}+\beta \frac{\left\{z f^{\prime}(z)\right\}^{\prime}}{f^{\prime}(z)}<\frac{1+z}{1-z}
\end{aligned}
$$

(1.5)

Let $\mathcal{A}(\alpha, \beta ; A, B)$ denotes the subclass of $\mathcal{A}(\alpha, \beta)$ consisting of the functions $g(z)=z+\sum_{n=2}^{\infty} b_{n} z^{n} \in \mathcal{A}$ and satisfying the condition

$(1-\alpha-\beta) f^{\prime}(z)+\alpha \frac{z f^{\prime}(z)}{f(z)}+\beta \frac{\left\{z f^{\prime}(z)\right\}^{\prime}}{f^{\prime}(z)} \prec \frac{1+\mathrm{Az}}{1+\mathrm{Bz}} ;-1 \leq$

$B \leq A \leq 1$

Let $\mathcal{A}(\alpha, \beta ; \delta)$ denotes the subclass of $\mathcal{A}(\alpha, \beta)$ consisting of the functions $g(z)=z+\sum_{n=2}^{\infty} b_{n} z^{n} \in \mathcal{A}$ and satisfying the condition

$$
(1-\alpha-\beta) f^{\prime}(z)+\alpha \frac{z f^{\prime}(z)}{f(z)}+\beta \frac{\left\{z f^{\prime}(z)\right\}^{\prime}}{f^{\prime}(z)}<\left(\frac{1+z}{1-z}\right)^{\delta} ; \delta>
$$

And if the subclass of $\mathcal{A}(\alpha, \beta)$ consisting of the functions $g(z)=z+\sum_{n=2}^{\infty} b_{n} z^{n} \in \mathcal{A}$ and satisfying the condition

$(1-\alpha-\beta) f^{\prime}(z)+\alpha \frac{z f^{\prime}(z)}{f(z)}+\beta \frac{\left\{z f^{\prime}(z)\right\}^{\prime}}{f^{\prime}(z)} \prec$

$\left(\frac{1+\mathrm{Az}}{1+\mathrm{Bz}}\right)^{\delta} ;-1 \leq B \leq A \leq 1 ; \delta>0$

is denoted by $\mathcal{A}(\alpha, \beta ; A, B ; \delta)$.

Here it is to be noted that
(i) $\quad \mathcal{A}(\alpha, \beta ; A, B ; 1)=\mathcal{A}(\alpha, \beta ; A, B)$
(ii) $\quad \mathcal{A}(\alpha, \beta ; 1,-1 ; \delta)=\mathcal{A}(\alpha, \beta ; \delta)$
(iii) $\quad \mathcal{A}(\alpha, \beta ; 1,-1)=\mathcal{A}(\alpha, \beta)$
(iv) $\quad \mathcal{A}(\alpha, \beta ; 1)=\mathcal{A}(\alpha, \beta)$
(v) $\quad \mathcal{A}(1,0)=S^{*}$
(vi) $\quad \mathcal{A}(0,1)=\mathcal{K}$ 


\section{Available online at www.ijrat.org}

Symbol $\prec$ stands for subordination, which we define as follows:

PRINCIPLE OF SUBORDINATION: Let $f(z)$ and $F(z)$ are two functions which are analytic in $\mathbb{E}$. Then $f(z)$ is called subordinate to $F(z)$ in $\mathbb{E}$, if there exists a function $w(z)$ analytic in $\mathbb{E}$ satisfying the conditions $w(0)=0$ and $\quad|w(z)|<1 \quad$ such that $f(z)=$ $F(w(z)) ; z \in \mathbb{E}$ and we write it as $f(z) \prec F(z)$.

Let $\mathcal{U}$ denote the class of analytic bounded functions of the form

$w(z)=\sum_{n=1}^{\infty} d_{n} z^{n}, w(0)=0,|w(z)|<1$.

(1.9)

It is known that $\left|d_{1}\right| \leq 1$ and $\left|d_{2}\right| \leq 1-\left|d_{1}\right|^{2}$.

\section{MAIN RESULTS AND DISCUSSION}

Theorem 2.1 if $\mathbf{f}(\mathbf{z}) \in \mathcal{A}(\alpha, \beta)$, then the result

$\left|\mathrm{a}_{2}-\mu \mathrm{a}_{2}^{2}\right| \leq\left\{\begin{array}{l}\left(\frac{2 \delta}{2-\alpha}\right)^{2}\left\{\frac{\left(4-2 \alpha+\alpha^{2}+8 \beta\right)}{2(3-\alpha+3 \beta)}-\mu\right\}, \text { if } \mu \leq \frac{\delta\left(4-2 \alpha+\alpha^{2}+8 \beta\right)-(2-\alpha)^{2}}{2 \delta(3-\alpha+3 \beta)} \\ \frac{2 \delta}{(3-\alpha+3 \beta)}, \text { if } \frac{\delta\left(4-2 \alpha+\alpha^{2}+8 \beta\right)-(2-\alpha)^{2}}{2 \delta(3-\alpha+3 \beta)} \leq \mu \leq \frac{\delta\left(4-2 \alpha+\alpha^{2}+8 \beta\right)+(2-\alpha)^{2}}{2 \delta(3-\alpha+3 \beta)} \\ \left(\frac{2 \delta}{2-\alpha}\right)^{2}\left\{\mu-\frac{\left(4-2 \alpha+\alpha^{2}+8 \beta\right)}{2(3-\alpha+3 \beta)}\right\}, \text { if } \mu \geq \frac{\delta\left(4-2 \alpha+\alpha^{2}+8 \beta\right)+(2-\alpha)^{2}}{2 \delta(3-\alpha+3 \beta)}\end{array}\right.$

Is sharp.

Proof: By the definition of $\mathcal{A}(\alpha, \beta)$, we have

$(1-\alpha-\beta) \mathrm{f}^{\prime}(\mathrm{z})+\alpha \frac{\mathrm{f}^{\prime}(\mathrm{z})}{\mathrm{f}(\mathrm{z})}+\beta \frac{\left\{\mathrm{zf}^{\prime}(\mathrm{z})\right\}^{\prime}}{\mathrm{f}^{\prime}(\mathrm{z})}<\left(\frac{1+z}{1-z}\right)^{\delta}$

On expanding (2.4), we have

$1+(2-\alpha) a_{2} z+\left\{(3-\alpha+3 \beta) a_{3}-(\alpha+4 \beta) a_{2}^{2}\right\} z^{2}+---=1+2 \delta c_{1} z+2 \delta\left(c_{2}+\delta c_{2}^{2}\right) z^{2}+---$

From the equation (2.5), we have

$\left\{\begin{array}{c}a_{2}=\frac{2 \delta}{2-\alpha} c_{1} \\ a_{3}=\frac{2 \delta}{(3-\alpha+3 \beta)}\left[c_{2}+\left\{\frac{\delta\left(4-2 \alpha+\alpha^{2}+8 \beta\right)}{(2-\alpha)^{2}}\right\} c_{1}^{2}\right]\end{array}\right.$

Using (2.6), we have

$\mathrm{a}_{3}-\mu \mathrm{a}_{2}^{2}=\frac{2 \delta}{(3-\alpha+3 \beta)} c_{2}+\left\{\frac{2 \delta^{2}\left(4-2 \alpha+\alpha^{2}+8 \beta\right)}{(3-\alpha+3 \beta)(2-\alpha)^{2}}-\frac{4 \delta^{2} \mu}{(2-\alpha)^{2}}\right\} c_{1}^{2}$

This leads to

$\left|\mathrm{a}_{3}-\mu \mathrm{a}_{2}^{2}\right| \leq \frac{2 \delta}{(3-\alpha+3 \beta)}+\left\{\left|\frac{2 \delta^{2}\left(4-2 \alpha+\alpha^{2}+8 \beta\right)}{(3-\alpha+3 \beta)(2-\alpha)^{2}}-\frac{4 \delta^{2} \mu}{(2-\alpha)^{2}}\right|-\frac{2 \delta}{(3-\alpha+3 \beta)}\right\}\left|c_{1}^{2}\right|$

Now two cases arise

CaseI: when $\mu \leq \frac{\left(4-2 \alpha+\alpha^{2}+8 \beta\right)}{2(3-\alpha+3 \beta)}$, then inequality (2.7)leads to

$\left|\mathrm{a}_{3}-\mu \mathrm{a}_{2}^{2}\right| \leq \frac{2 \delta}{(3-\alpha+3 \beta)}+\left\{\frac{2 \delta^{2}\left(4-2 \alpha+\alpha^{2}+8 \beta\right)}{(3-\alpha+3 \beta)(2-\alpha)^{2}}-\frac{4 \delta^{2} \mu}{(2-\alpha)^{2}}-\frac{2 \delta}{(3-\alpha+3 \beta)}\right\}\left|c_{1}^{2}\right|$

Which feather leads to

$\left|\mathrm{a}_{3}-\mu \mathrm{a}_{2}^{2}\right| \leq \frac{2 \delta}{(3-\alpha+3 \beta)}+\frac{2 \delta}{(2-\alpha)^{2}}\left\{\frac{\delta\left(4-2 \alpha+\alpha^{2}+8 \beta\right)-(2-\alpha)^{2}}{(3-\alpha+3 \beta)}-2 \delta \mu\right\}\left|c_{1}^{2}\right|$

Under this case, two sub cases arise

Subcase I(a): when $\mu \leq \frac{\delta\left(4-2 \alpha+\alpha^{2}+8 \beta\right)-(2-\alpha)^{2}}{2 \delta(3-\alpha+3 \beta)}$, then inequality (2.8)leads to

$\left|\mathrm{a}_{3}-\mu \mathrm{a}_{2}^{2}\right| \leq \frac{2 \delta}{(3-\alpha+3 \beta)}+\frac{2 \delta}{(2-\alpha)^{2}}\left\{\frac{\delta\left(4-2 \alpha+\alpha^{2}+8 \beta\right)-(2-\alpha)^{2}}{(3-\alpha+3 \beta)}-2 \delta \mu\right\}$

$\left|\mathrm{a}_{3}-\mu \mathrm{a}_{2}^{2}\right| \leq\left(\frac{2 \delta}{2-\alpha}\right)^{2}\left\{\frac{\left(4-2 \alpha+\alpha^{2}+8 \beta\right)}{2(3-\alpha+3 \beta)}-\mu\right\}$

Subcase I (b): when $\mu \geq \frac{\delta\left(4-2 \alpha+\alpha^{2}+8 \beta\right)-(2-\alpha)^{2}}{2 \delta(3-\alpha+3 \beta)}$, then inequality (2.8)leads to 
$\left|\mathrm{a}_{3}-\mu \mathrm{a}_{2}^{2}\right| \leq \frac{2 \delta}{(3-\alpha+3 \beta)}$

CaseII: when $\mu \geq \frac{\left(4-2 \alpha+\alpha^{2}+8 \beta\right)}{2(3-\alpha+3 \beta)}$, then inequality (2.7)leads to

$\left|\mathrm{a}_{3}-\mu \mathrm{a}_{2}^{2}\right| \leq \frac{2 \delta}{(3-\alpha+3 \beta)}+\left\{\frac{4 \delta^{2} \mu}{(2-\alpha)^{2}}-\frac{\delta\left(4-2 \alpha+\alpha^{2}+8 \beta\right)+(2-\alpha)^{2}}{(3-\alpha+3 \beta)(2-\alpha)^{2}}\right\}\left|c_{1}^{2}\right|$

Now again two subcases arise under the case II.

Subcase II(a): when $\mu \leq \frac{\delta\left(4-2 \alpha+\alpha^{2}+8 \beta\right)+(2-\alpha)^{2}}{2 \delta(3-\alpha+3 \beta)}$, we have

$\left|\mathrm{a}_{3}-\mu \mathrm{a}_{2}^{2}\right| \leq \frac{2 \delta}{(3-\alpha+3 \beta)}$

Subcase II(b): when $\mu \geq \frac{\delta\left(4-2 \alpha+\alpha^{2}+8 \beta\right)+(2-\alpha)^{2}}{2 \delta(3-\alpha+3 \beta)}$, we have

$\left|\mathrm{a}_{3}-\mu \mathrm{a}_{2}^{2}\right| \leq\left(\frac{2 \delta}{2-\alpha}\right)^{2}\left\{\mu-\frac{\left(4-2 \alpha+\alpha^{2}+8 \beta\right)}{2(3-\alpha+3 \beta)}\right\}$

Combining the results of subcase I(b) and subcase II(a), we get

$\left|\mathrm{a}_{3}-\mu \mathrm{a}_{2}^{2}\right| \leq \frac{2 \delta}{(3-\alpha+3 \beta)}$,if $\frac{\delta\left(4-2 \alpha+\alpha^{2}+8 \beta\right)-(2-\alpha)^{2}}{2 \delta(3-\alpha+3 \beta)} \leq \mu \leq \frac{\delta\left(4-2 \alpha+\alpha^{2}+8 \beta\right)+(2-\alpha)^{2}}{2 \delta(3-\alpha+3 \beta)}$

This completes the theorem. The result are sharp.

Extremal function for inequalities (2.1) and (2.3) will be as follows:

$f_{1}(z)=z\left(1+\frac{q-2 p}{\sqrt{q}} z\right)^{\frac{q}{q-2 p}}$

where $\mathrm{p}=\left(\frac{2 \delta}{2-\alpha}\right)^{2}\left\{\frac{\left(4-2 \alpha+\alpha^{2}+8 \beta\right)}{2(3-\alpha+3 \beta)}\right\}$ and $\mathrm{q}=\left(\frac{2 \delta}{2-\alpha}\right)^{2}$

Extremal function for inequalities (2.2) leads to

$f_{2}(z)=z(1+2 z)^{\frac{\delta}{(3-\alpha+3 \beta)}}$

Theorem 3.1 if $\mathbf{f}(\mathbf{z}) \in \mathcal{A}(\alpha, \beta)$, then the result

$$
\left|\mathrm{a}_{3}-\mu \mathrm{a}_{2}^{2}\right| \leq\left\{\begin{array}{c}
\delta^{2}\left(\frac{\mathrm{A}-\mathrm{B}}{2-\alpha}\right)^{2}\left\{\frac{\{\delta(\mathrm{A}-\mathrm{B})-(\mathrm{A}+\mathrm{B})\}(2-\alpha)^{2}+2 \delta(\alpha+4 \beta)(\mathrm{A}-\mathrm{B})}{2 \delta(\mathrm{A}-\mathrm{B})(3-\alpha+3 \beta)}-\mu\right\}, \\
\text { if } \mu \leq \frac{\{\delta(\mathrm{A}-\mathrm{B})-(\mathrm{A}+\mathrm{B})-2\}(2-\alpha)^{2}+2 \delta(\alpha+4 \beta)(\mathrm{A}-\mathrm{B})}{2 \delta(\mathrm{A}-\mathrm{B})(3-\alpha+3 \beta)} \\
\frac{(\mathrm{A}-\mathrm{B}) \delta}{(3-\alpha+3 \beta)}, \text { if } \frac{\{\delta(\mathrm{A}-\mathrm{B})-(\mathrm{A}+\mathrm{B})-2\}(2-\alpha)^{2}+2 \delta(\alpha+4 \beta)(\mathrm{A}-\mathrm{B})}{2 \delta(\mathrm{A}-\mathrm{B})(3-\alpha+3 \beta)} \\
\mu \leq \frac{\{\delta(\mathrm{A}-\mathrm{B})-(\mathrm{A}+\mathrm{B})+2\}(2-\alpha)^{2}+2 \delta(\alpha+4 \beta)(\mathrm{A}-\mathrm{B})}{2 \delta(\mathrm{A}-\mathrm{B})(3-\alpha+3 \beta)} \\
\delta^{2}\left(\frac{\mathrm{A}-\mathrm{B}}{2-\alpha}\right)^{2}\left\{\mu-\frac{\{\delta(\mathrm{A}-\mathrm{B})-(\mathrm{A}+\mathrm{B})\}(2-\alpha)^{2}+2 \delta(\alpha+4 \beta)(\mathrm{A}-\mathrm{B})}{2 \delta(\mathrm{A}-\mathrm{B})(3-\alpha+3 \beta)}\right\} \\
\text { if } \mu \leq \frac{\{\delta(\mathrm{A}-\mathrm{B})-(\mathrm{A}+\mathrm{B})+2\}(2-\alpha)^{2}+2 \delta(\alpha+4 \beta)(\mathrm{A}-\mathrm{B})}{2 \delta(\mathrm{A}-\mathrm{B})(3-\alpha+3 \beta)}
\end{array},\right.
$$

is sharp.

Proof: By the definition of $\mathcal{A}(\alpha, \beta)$, we have

$(1-\alpha-\beta) \mathrm{f}^{\prime}(\mathrm{z})+\alpha \frac{\mathrm{f}^{\prime}(\mathrm{z})}{\mathrm{f}(\mathrm{z})}+\beta \frac{\left\{\mathrm{zf}^{\prime}(\mathrm{z})\right\}^{\prime}}{\mathrm{f}^{\prime}(\mathrm{z})} \prec\left(\frac{1+A z}{1+B z}\right)^{\delta}$

On expanding (3.4), we have

$1+(2-\alpha) a_{2} z+\left\{(3-\alpha+3 \beta) a_{3}-(\alpha+4 \beta) a_{2}^{2}\right\} z^{2}+---=1+(A-B) \delta c_{1} z+\left[\delta(A-B) c_{2}+\frac{\delta}{2}(A-B)\{\delta(A-\right.$ $\left.B)-(A+B)\} c_{1}^{2}\right] z^{2}+---$ 
From the equation (3.5), we have

$$
\left\{\begin{array}{c}
a_{2}=\frac{\delta(A-B)}{2-\alpha} c_{1} \\
a_{3}=\frac{\delta(A-B)}{(3-\alpha+3 \beta)}\left[c_{2}+\left[\frac{1}{2}\{\delta(A-B)-(A+B)\}+\frac{(\alpha+4 \beta) \delta(A-B)}{(2-\alpha)^{2}}\right] c_{1}^{2}\right]
\end{array}\right.
$$

Using (3.6), we have

$\mathrm{a}_{3}-\mu \mathrm{a}_{2}^{2}=\frac{\delta(A-B)}{(3-\alpha+3 \beta)} c_{2}+\left[\frac{\delta(A-B)}{(3-\alpha+3 \beta)}\left\{\frac{1}{2}\{\delta(A-B)-(A+B)\}+\frac{(\alpha+4 \beta) \delta(A-B)}{(2-\alpha)^{2}}\right\}-\frac{(\mathrm{A}-\mathrm{B})^{2} \delta^{2} \mu}{(2-\alpha)^{2}}\right] c_{1}^{2}$

This leads to

$$
\begin{aligned}
&\left|\mathrm{a}_{3}-\mu \mathrm{a}_{2}^{2}\right| \leq \frac{\delta(A-B)}{(3-\alpha+3 \beta)} \\
& \quad+\left[\frac{\delta(A-B)}{2(2-\alpha)^{2}}\left\{\left|\frac{\{\delta(A-B)-(A+B)\}(2-\alpha)^{2}+2(\alpha+4 \beta) \delta(A-B)}{(3-\alpha+3 \beta)}-2 \delta(A-B) \mu\right|\right\}\right. \\
&\left.\quad-\frac{\delta(A-B)}{(3-\alpha+3 \beta)}\right]\left|c_{1}^{2}\right|
\end{aligned}
$$

Now two cases arise

CaseI: when $\mu \leq \frac{\{\delta(A-B)-(A+B)\}(2-\alpha)^{2}+2(\alpha+4 \beta) \delta(A-B)}{2 \delta(A-B)(3-\alpha+3 \beta)}$, then inequality (3.7)leads to $\left|\mathrm{a}_{3}-\mu \mathrm{a}_{2}^{2}\right| \leq \frac{\delta(A-B)}{(3-\alpha+3 \beta)}$

$$
\begin{aligned}
& +\left[\frac{\delta(A-B)}{2(2-\alpha)^{2}}\left\{\left|\frac{\{\delta(A-B)-(A+B)\}(2-\alpha)^{2}+2(\alpha+4 \beta) \delta(A-B)}{(3-\alpha+3 \beta)}-2 \delta(A-B) \mu\right|\right\}\right. \\
& \left.-\frac{\delta(A-B)}{(3-\alpha+3 \beta)}\right]\left|c_{1}^{2}\right|
\end{aligned}
$$

Which feather leads to

$$
\begin{aligned}
&\left|\mathrm{a}_{3}-\mu \mathrm{a}_{2}^{2}\right| \leq \frac{\delta(A-B)}{(3-\alpha+3 \beta)} \\
& \quad+\frac{\delta(A-B)}{2(2-\alpha)^{2}}\left\{\mid \frac{\{\delta(A-B)-(A+B)-2\}(2-\alpha)^{2}+2(\alpha+4 \beta) \delta(A-B)}{(3-\alpha+3 \beta)}\right. \\
&\quad-2 \delta(A-B) \mu \mid\}\left|c_{1}^{2}\right|
\end{aligned}
$$

Under this case, two sub cases arise

Subcase I(a): when $\mu \leq \frac{\{\delta(A-B)-(A+B)-2\}(2-\alpha)^{2}+2(\alpha+4 \beta) \delta(A-B)}{2 \delta(A-B)(3-\alpha+3 \beta)}$, then inequality (3.8)leads to $\left|\mathrm{a}_{3}-\mu \mathrm{a}_{2}^{2}\right| \leq \frac{\delta(A-B)}{(3-\alpha+3 \beta)}+\frac{\delta(A-B)}{2(2-\alpha)^{2}}\left\{\frac{\{\delta(A-B)-(A+B)-2\}(2-\alpha)^{2}+2(\alpha+4 \beta) \delta(A-B)}{2 \delta(A-B)(3-\alpha+3 \beta)}-2 \delta(A-B) \mu\right\}$ $\left|\mathrm{a}_{3}-\mu \mathrm{a}_{2}^{2}\right| \leq \frac{\delta^{2}(A-B)^{2}}{(2-\alpha)^{2}}\left\{\frac{\{\delta(A-B)-(A+B)\}(2-\alpha)^{2}+2(\alpha+4 \beta) \delta(A-B)}{2 \delta(A-B)(3-\alpha+3 \beta)}-\mu\right\}$

Subcase $\mathbf{I}(\mathbf{b})$ : when $\mu \geq \frac{\{\delta(A-B)-(A+B)-2\}(2-\alpha)^{2}+2(\alpha+4 \beta) \delta(A-B)}{2 \delta(A-B)(3-\alpha+3 \beta)}$, then inequality (3.8)leads to $\left|\mathrm{a}_{3}-\mu \mathrm{a}_{2}^{2}\right| \leq \frac{(A-B) \delta}{(3-\alpha+3 \beta)}$

CaseII: when $\mu \geq \frac{\{\delta(A-B)-(A+B)\}(2-\alpha)^{2}+2(\alpha+4 \beta) \delta(A-B)}{2 \delta(A-B)(3-\alpha+3 \beta)}$, then inequality (3.7)leads to

$\left|\mathrm{a}_{3}-\mu \mathrm{a}_{2}^{2}\right| \leq \frac{\delta(A-B)}{(3-\alpha+3 \beta)}$

$$
\begin{aligned}
& +\frac{\delta(A-B)}{2(2-\alpha)^{2}}\{\mid 2 \delta(A-B) \mu \\
& \left.-\frac{\{\delta(A-B)-(A+B)+2\}(2-\alpha)^{2}+2(\alpha+4 \beta) \delta(A-B)}{(3-\alpha+3 \beta)} \mid\right\}\left|c_{1}^{2}\right|
\end{aligned}
$$


Now again two subcases arise under the case II.

Subcase II (a): when $\mu \leq \frac{\{\delta(A-B)-(A+B)+2\}(2-\alpha)^{2}+2(\alpha+4 \beta) \delta(A-B)}{2 \delta(A-B)(3-\alpha+3 \beta)}$, then inequality (3.9)leads to $\left|\mathrm{a}_{3}-\mu \mathrm{a}_{2}^{2}\right| \leq \frac{\delta(A-B)}{(3-\alpha+3 \beta)}$

Subcase II (b): when $\mu \geq \frac{\{\delta(A-B)-(A+B)+2\}(2-\alpha)^{2}+2(\alpha+4 \beta) \delta(A-B)}{2 \delta(A-B)(3-\alpha+3 \beta)}$, then inequality (3.9)leads to

$\left|\mathrm{a}_{3}-\mu \mathrm{a}_{2}^{2}\right| \leq \frac{\delta^{2}(A-B)^{2}}{(2-\alpha)^{2}}\left\{\mu-\frac{\{\delta(A-B)-(A+B)\}(2-\alpha)^{2}+2(\alpha+4 \beta) \delta(A-B)}{2 \delta(A-B)(3-\alpha+3 \beta)}\right\}$

Combining the results of subcase I(b) and subcase II(a), we get

$$
\begin{gathered}
\left|\mathrm{a}_{3}-\mu \mathrm{a}_{2}^{2}\right| \leq \frac{\delta(A-B)}{(3-\alpha+3 \beta)}, \text { if } \frac{\{\delta(A-B)-(A+B)-2\}(2-\alpha)^{2}+2(\alpha+4 \beta) \delta(A-B)}{2 \delta(A-B)(3-\alpha+3 \beta)} \leq \mu \\
\leq \frac{\{\delta(A-B)-(A+B)+2\}(2-\alpha)^{2}+2(\alpha+4 \beta) \delta(A-B)}{2 \delta(A-B)(3-\alpha+3 \beta)}
\end{gathered}
$$

This completes the theorem. The result are sharp.

Extremal function for inequalities (3.1) and (3.3) will be as follows:

$f_{1}(z)=z\left(1+\frac{s-2 r}{\sqrt{s}} z\right)^{\frac{s}{s-2 r}}$

where $\mathrm{r}=\frac{\delta^{2}(A-B)^{2}}{(2-\alpha)^{2}}\left\{\frac{\{\delta(A-B)-(A+B)\}(2-\alpha)^{2}+2(\alpha+4 \beta) \delta(A-B)}{2 \delta(A-B)(3-\alpha+3 \beta)}\right\} \quad$ and $\mathrm{s}=\frac{\delta^{2}(A-B)^{2}}{(2-\alpha)^{2}}$

Extremal function for inequalities (3.2) leads to

$f_{2}(z)=\{1+(A-B) Z\}^{\frac{\delta}{(3-\alpha+3 \beta)}}$

\section{CONCLUDING REMARKS}

If we take $\mathrm{A}=1$ and $\mathrm{B}=-1$ in the result of theorem 3.1 , we get the result of theorem 2.1, therefore our result for the theorem 3.1 reduces to the result of the theorem 2.1. Hence theorem 3.1 is the generalization of theorem 2.1. And the results are sharp.

\section{REFERENCES}

[1] Kunle Oladeji Bablola,"The fifth andsixth coefficient of $\alpha$-close-to-convexfunction," Kragujevac J. Math.32,5-12,2009

[2] K.Lowner,"Uber monotone Matrixfunktionen" Math. Z 38,177-216,1934

[3] L.Bieberbach,"Uber Einige Extermal Probleme im Gebiete der Konformen Abbildung," Math. 77,153172,1916
[4] L. Bieberbach,"Uberdie Koeffizientem derjenigem Potenzreihen, welche eine Schlithe Abblidung des Einheitskrises Vermittelen," Preuss. Akad. Wiss Sitzungsb. 940-955,1916

[5] M.Fekete and G.Szego," Eine Bemerkung Uber ungerade Schlichte Funktionen," J.London Math. Soc. 8,85-89,1933

[6] P.N. Chhichra,"New Subclasses of the class of close to convex functions" Procedure of American Mathematical Society, 62,37-43,1973

\section{*Corresponding Author:}

Lokendra Kumawat (Research scholar) Department of Mathematics \& Statistics Mohanlal Sukhadia University, Udaipur,(Raj) Email:- lokendrakumawat@yahoo.co.in 